\title{
A Genome-Wide Association Study Identifies UTRN Gene Polymorphism for Restless Legs Syndrome in a Korean Population
}

\author{
Chul-Hyun Cho ${ }^{1 *}$, Ji-Hye Choi ${ }^{2 *}$, Seung-Gul Kang ${ }^{3}$, Ho-Kyoung Yoon ${ }^{1}$, Young-Min Park ${ }^{4}$, \\ Joung-Ho Moon ${ }^{1}$, Ki-Young Jung ${ }^{5}$, Jin-Kyu Han ${ }^{6}$, Hong-Bum Shin ${ }^{7}$, Hyun Ji Noh ${ }^{8}$, \\ Yong Seo Koo ${ }^{9}$, Leen Kim ${ }^{1}$, Hyun Goo Woo ${ }^{2}$, and Heon-Jeong Lee ${ }^{1 凶}$ \\ ${ }^{1}$ Department of Psychiatry, Korea University College of Medicine, Seoul, Republic of Korea \\ ${ }^{2}$ Department of Physiology, Ajou University School of Medicine, Suwon, Republic of Korea \\ ${ }^{3}$ Department of Psychiatry, School of Medicine, Gachon University, Incheon, Republic of Korea \\ ${ }^{4}$ Department of Psychiatry, Inje University, Ilsan Paik Hospital, Goyang, Republic of Korea \\ ${ }^{5}$ Department of Neurology, Seoul University College of Medicine, Seoul, Republic of Korea \\ ${ }^{6}$ Seoul Sleep Center, Seoul, Republic of Korea \\ ${ }^{7}$ Kosleep Clinic, Seoul, Republic of Korea \\ ${ }^{8}$ Broad Institute of MIT and Harvard, Cambridge, MA, USA \\ ${ }^{9}$ Department of Neurology, Korea University College of Medicine, Seoul, Republic of Korea
}

Objective Restless legs syndrome (RLS) is a highly heritable and common neurological sensorimotor disease disturbing sleep. The objective of study was to investigate significant gene for RLS by performing GWA and replication study in a Korean population.

Methods We performed a GWA study for RLS symptom group $(n=325)$ and non-RLS group $(n=2,603)$ from the Korea Genome Epidemiology Study. We subsequently performed a replication study in RLS and normal controls (227 RLS and 229 controls) to confirm the present GWA study findings as well as previous GWA study results.

Results In the initial GWA study of RLS, we observed an association of rs 11645604 (OR=1.531, $\left.\mathrm{p}=1.18 \times 10^{-6}\right)$ in $\mathrm{MPHOSPH6}$ on chromosome 16q23.3, rs1918752 (OR=0.6582, $\left.\mathrm{p}=1.93 \times 10^{-6}\right)$ and $\mathrm{rs} 9390170\left(\mathrm{OR}=0.6778, \mathrm{p}=7.67 \times 10^{-6}\right)$ in $U T R N$ on chromosome $6 \mathrm{q} 24$. From the replication samples, we found $\mathrm{rs} 9390170$ in UTRN ( $\mathrm{p}=0.036)$ and $\mathrm{rs} 3923809$ and rs9296249 in BTBD9 ( $\mathrm{p}=0.045$, $\mathrm{p}=0.046$, respectively) were significantly associated with RLS. Moreover, we found the haplotype polymorphisms of rs9357271, rs3923809, and rs9296249 (overall $\mathrm{p}=5.69 \times 10^{-18}$ ) in BTBD9 was associated with RLS.

Conclusion From our sequential GWA and replication study, we could hypothesize rs 9390170 polymorphism in UTRN is a novel genetic marker for susceptibility to RLS. Regarding with utrophin, which is encoded by UTRN, is preferentially expressed in the neuromuscular synapse and myotendinous junctions, we speculate that utrophin is involved in RLS, particularly related to the neuromuscular aspects.

Psychiatry Investig 2017;14(6):830-838

Key Words Restless legs syndrome, Genome-wide association study, Replication study, Utrophin, UTRN.

\section{INTRODUCTION}

Restless legs syndrome (RLS), also known as Willis-Ekbom disease (WED), is a common neurological sensorimotor disease that disables sensory and motor elements. RLS is diagnosed by: 1) an urge to move the legs usually but not always

\footnotetext{
Received: January 2, 2017 Revised: August 7, 2017 Accepted: August 15, 2017 Available online: October 17, 2017

$\triangle$ Correspondence: Hyun Goo Woo, MD, PhD

Department of Physiology, Ajou University School of Medicine, 164 Worldcup-ro, Yeongtong-gu, Suwon 16499, Republic of Korea

Tel: +82-31-219-5045, Fax: +82-31-219-5049, E-mail: hg@ajou.ac.kr

$\triangle$ Correspondence: Heon-Jeong Lee, $\mathrm{MD}, \mathrm{PhD}$

Department of Psychiatry, Anam Hospital, Korea University College of Medicine, 73 Inchon-ro, Seongbuk-gu, Seoul 02841, Republic of Korea

Tel: +82-2-920-5815, Fax: +82-2-929-7679, E-mail: leehjeong@korea.ac.kr

*These authors contributed equally to this work.

@ This is an Open Access article distributed under the terms of the Creative Commons Attribution Non-Commercial License (http://creativecommons.org/licenses/by-nc/4.0) which permits unrestricted non-commercial use, distribution, and reproduction in any medium, provided the original work is properly cited.
} 
accompanied by or felt to be caused by uncomfortable and unpleasant sensations in the legs; 2) the urge to move the legs and any accompanying unpleasant sensations begin or worsen during periods of rest or inactivity such as lying down or sitting; 3 ) the urge to move the legs and any accompanying unpleasant sensations are partially or totally relieved by movement, such as walking or stretching, at least as long as the activity continues; 4) the urge to move the legs and any accompanying unpleasant sensations during rest or inactivity only occur or are worse in the evening or night than during the day; and 5) the occurrence of the above features is not solely accounted for as symptoms primary to another medical or behavioral condition (e.g., myalgia, venous stasis, leg edema, arthritis, leg cramps, positional discomfort, habitual foot tapping). ${ }^{1}$ As a result, patients with RLS often suffer from sleep initiation and maintenance disorders. A recent study in North American and Western European populations reported that general prevalence rates of RLS ranged from $4 \%$ to $29 \%$ in adults. ${ }^{2}$ The prevalence of RLS increases with age, and RLS is more common among women than men. ${ }^{3}$ RLS has a negative impact on physical as well as mental health, resulting in poor quality of life. In spite of these observations, the pathogenesis of RLS is not clearly understood. RLS is known to be associated with iron deficiency, and RLS pathologic studies have reported reduced brain and cerebrospinal fluid iron and iron-associated proteins. ${ }^{4}$ Genetic predisposition and dopaminergic dysfunction are also thought to be underlying factors in RLS. ${ }^{5}$ RLS is relieved by administration of dopamine agonists, which are currently the best-studied therapeutic regimen. Dopaminergic agents, which were developed for Parkinson's disease, have effect on RLS via unknown mode of action. ${ }^{6}$ Other treatments of RLS are also aimed at reducing symptoms and include opioids, sedative hypnotics, anticonvulsants, and benzodiazepines. ${ }^{7}$ Unfortunately, there is currently no treatment of choice for curing RLS. Pharmacological, neuroimaging, and neurophysiological studies suggest that the essential symptoms originate in the central nervous system, yet the underlying mechanism remains unclear. ${ }^{8}$

A familial inclination has been reported, particularly in early-onset RLS. ${ }^{9}$ More than $50 \%$ of RLS-affected subjects reported a family history of RLS, ${ }^{10}$ and the heritability of RLS was reported in large and extended multiplex pedigrees $\left(\mathrm{h}^{2}=\right.$ 0.6). ${ }^{11}$ The results of linkage analysis suggested six loci related to recessive or dominant inheritance; $12 \mathrm{q}, 14 \mathrm{q}, 9 \mathrm{p}, 2 \mathrm{q}, 20 \mathrm{p}$, and 19p (RLS1-6, respectively), but no specific genes. ${ }^{12}$ There were two noteworthy genome-wide association (GWA) studies showing an association of RLS with genetic components. Winkelmann et al. ${ }^{13}$ reported GWA study results showing genome-wide significant association between RLS and three genetic loci: one was on MEIS1 within chromosome 2p, one was on BTBD9 in chromosome $6 \mathrm{p}$, and one was on between $M A P 2 K 5$ and LBXCOR1 on chromosome 15q. The same group also reported that $P T P R D$ at 9p23-24 is associated with RLS through a GWA study. ${ }^{14}$ Stefansson et al. ${ }^{15}$ also reported GWA study results demonstrating an association between RLS and a locus on chromosome $6 \mathrm{p}$ in the fifth intron of BTBD9. Recent GWA studies of RLS identified novel susceptibility loci on 2 p14 and $16 \mathrm{q} 12.1 .^{16}$

Although there have been various genetic association studies of RLS from various ethnic groups, all of the GWA studies of RLS were performed only in Caucasian populations. Because there are possible ethnic differences in genetic influence, it would be advantageous to perform GWA studies of RLS in ethnically diverse populations.

We performed a GWA study of RLS to find susceptibility genes for RLS in Korean cohort samples, and tried to replicate the results from our study as well as the previous GWA studies in Caucasian populations in the independent clinical samples of RLS and non-RLS controls in a Korean population.

\section{METHODS}

\section{Subjects}

Subjects for the GWA study were collected from the Ansung and Ansan cohorts of the Korea Association Resource (KARE) project: 7,515 participants ranging in age from 40 to 69 years. These cohorts were investigated as part of the Korea Genome Epidemiology Study (KoGES). Epidemiological information of the cohorts contains more than 260 traits, including demographic information, medical and family disease histories, health condition, and lifestyle such as sleep pattern. ${ }^{17}$ We focused on RLS symptoms based on questionnaire results and medical history that may be related to RLSlike symptoms such as diabetes mellitus, thyroid disease, kidney disease, and spinal cord disease. Written informed consent and ethics committee approval had already been obtained in the process of establishing the KoGES. ${ }^{18}$ The RLS clinical samples for the replication study were collected from multiple centers including the Korea University Hospital Sleep Clinic and two private sleep clinics in Seoul, South Korea. All the subjects visited sleep clinics due to insomnia. Sleep specialists (HJL, KYJ, JKH, or HBS) made diagnoses based on patients' signs and symptoms, medical and family histories, physical exams, and test results. The sleep specialists ruled out other conditions that have symptoms similar to those of RLS. Sexand age-matched healthy controls were selected based on RLS symptom evaluation and medical and family histories. Written informed consent from the patients and approval from the Institutional Review Board (IRB) of Korea University Hospital were obtained in the process of establishing the rep- 
lication study (IRB No. IEC107014).

\section{Genotyping and quality control}

Genomic DNAs for the GWA study were extracted from peripheral blood obtained from Ansung and Ansan cohort participants for genotyping with the Affymetrix GenomeWide Human SNP array 5.0. A total of 7,515 samples were genotyped, leading to an initial identification of 500,568 SNPs. An exclusion process was subsequently performed based on the quality control results such as high missing genotype call rate $(>4 \%)$, high heterozygosity $(>30 \%)$, gender inconsistencies, individuals who had developed any type of cancer, and higher computed average pairwise identity-by-state values than those estimated from first-degree relatives of Korean sibpair samples $(>0.80)$. In addition, markers with high missing gene call rate $(>5 \%)$, significant deviation from Hardy-Weinberg equilibrium $\left(\mathrm{p}<1 \times 10^{-6}\right)$, or low minor allele frequency $(<0.01)$ were excluded for quality control. After the quality control process, a total of 352,228 markers in 6,675 subjects remained.

A total of 467 subjects were genotyped for the replication study of GWA study findings. Blood was collected in 5-mL EDTA vacutainers (REF 367525, BD, Plymouth, UK) from study participants and stored at $-80^{\circ} \mathrm{C}$ until genotyping. Genomic DNA was extracted using the Blood DNA Purification Kit (CMB0115, Cosmo Genetech Co. Ltd., Seoul, Korea). After quality assessment with the NanoDrop spectrophotometer (ND-1000, NanoDrop Technologies, Wilmington, DE, USA) and polymerase chain reaction (PCR), $250 \mathrm{ng}$ of each DNA were genotyped by high resolution melting (HRM) analysis using a real-time PCR system with SsoFast EvaGreen Supermix (172-5205AP, Bio-Rad Laboratories, Hercules, CA, USA) and appropriate primer pairs for each SNP. The HardyWeinberg equilibrium test was performed to assess the goodness of fit of the data.

\section{Phenotypes}

We identified the RLS symptom group and non-RLS group for GWA. Among the 6,675 individuals remaining after quality control, the case and control groups were identified based on answers to a questionnaire regarding RLS symptoms. Individuals were asked four RLS-related questions based on the following situations: 1) an urge to move the legs, usually accompanied or caused by uncomfortable and unpleasant sensations in the legs, 2) the urge to move or unpleasant sensations beginning or worsening during periods of rest or inactivity such as lying or sitting, 3) the urge to move or unpleasant sensations are partially or totally relieved by movement, such as walking or stretching, at least as long as the activity continues, and 4) the urge to move or unpleasant sensations are worse in the evening or nighttime than during the day or only occur in the evening or night. ${ }^{6}$ Responders of the questionnaires were divided into the RLS symptom group and non-RLS group, whereas non-responders were categorized as the missing group. Subjects with medical conditions which may show symptoms similar to RLS, such as diabetes mellitus, thyroid disease, kidney disease, or spinal disease, were also categorized in the missing group. Because the numbers $(n=127)$ of individuals who answered 'yes' to all four questionnaire items were not sufficiently large to perform an analysis, individuals who answered 'yes' to at least the first question 1) of four questionnaires were assigned to the RLS symptom group. The first question of the above four is essential for diagnosis of RLS, and the questions 2-4) can be answered after answering the first question. Individuals who answered 'no' to all four questions were assigned to the non-RLS group. There were 325 subjects in the RLS symptom group, 2,603 subjects in the non-RLS group, and 3,747 subjects in the missing group within the present GWA study.

Our replication study samples were consistent with RLS clinical case and non-RLS matched controls. All the RLS cases and controls were assessed using the International Restless Legs Syndrome Study Group diagnostic criteria. RLS subjects with medical history that may cause RLS-like symptoms similar to those above were excluded from sample collection in the replication study. Finally, there were 227 subjects in the RLS cases and 229 control subjects.

\section{Association analyses}

To infer the genotypes for missing markers, the data were imputed with HapMap3 (release \#2, Feb 2009) reference panel by using IMPUTE2 software. ${ }^{19}$ After SNP imputation, we performed a logistic regression analysis between the case and control groups after adjustment for sex and age using the PLINK software package. ${ }^{20}$ Adaptive permutation was performed to assess the empirical significance of the results with default parameters. Manhattan plot was generated by Haploview software (ver. 4.2; Daly Lab at the Broad Institute, Cambridge, MA, USA).

Linkage disequilibrium (LD) blocks were identified from 1,000 Genomes Phase3 JPT+CHB [PMID:26432245] using Haploview software [PMID:15297300]. The proxy SNPs with regional recombination rates were assessed using SNAP [PMID: 18974171] (ver. 2.2; the Genomics Platform at the Broad Institute, Cambridge, MA, USA).

\section{Functional enrichment analysis}

For the genes harboring SNPs with significant association $(p<0.005)$, we performed functional enrichment analysis using Gene Ontology categories in DAVID software. ${ }^{21,22}$ The en- 
riched gene functions were indicated by the enrichment scores (ES) that indicates the biological significance of gene groups based on p-value for each enriched annotation terms.

\section{RESULTS}

\section{Genome-wide association study}

We performed a GWA of RLS symptom group vs. non-RLS group to identify SNPs that are associated with RLS in a Korean population. A total of 96 associated SNPs with $\mathrm{p}<10^{-4}$ were found in the present GWA study. Of these, rs11645604 $\left[\mathrm{OR}=1.531, \mathrm{p}=1.18 \times 10^{-6}\right.$ (permuted $\left.\left.\mathrm{p}=1.0 \times 10^{-6}\right)\right], \mathrm{rs} 1918752$ $\left[\mathrm{OR}=0.6582, \mathrm{p}=1.93 \times 10^{-6}\right.$ (permuted $\left.\left.\mathrm{p}=2.0 \times 10^{-6}\right)\right]$, and rs9390170 $\left[\mathrm{OR}=0.6778, \mathrm{p}=7.67 \times 10^{-6}\right.$ (permuted $\left.\left.\mathrm{p}=5.0 \times 10^{-6}\right)\right]$ showed the most significant associations (Figure 1). rs11645 604 is located within an intron of the gene encoding M-phase phosphoprotein 6 (MPHOSPH6) on chromosome 16q23.3. rs1918752 and rs9390170 are located within UTRN on chromosome 6q24.

Next, we identified two genomic regions of interest (ROIs) on chromosomes 6 and 16, defined as regions containing at least two SNPs with $\mathrm{p}<10^{-4}$ and adequate support for association (i.e. $\mathrm{p}<10^{-3}$ ) from surrounding SNPs within $100 \mathrm{~kb}$. From the identified ROI of the present GWA study, ROI1 and ROI2 contain the most associated genes, UTRN and MPHOSPH6, respectively (Supplementary Table 1 in the online-only Data Supplement). We estimated LD of both ROIs as described in Methods. As detailed in Figure 2A, support for the 6q24 region of the ROI1 came from a total of 30 SNPs spanning 164,659 $\mathrm{kb}$, many of which exhibited high levels of LD with the most significant SNP, rs1918752 on UTRN. As shown in Figure 2B, ROI2 at 16q23.3 region was supported from a total of 38 SNPs spanning 78,602 kb, many of which exhibited high levels of LD with the most significant SNP, rs11645604 on MPHOSPH6. In addition, two ROIs are located within or near blocks of high LD (Figure 2).

Next, we performed functional enrichment analysis to determine whether the associated SNPs show enrichment in certain functional pathways using 887 genes from SNPs associated with $\mathrm{p}<0.005$. As shown in Table 1 , the case-control analysis showed enrichment in nervous system development (enrichment scores, $\mathrm{ES}=4.19)$, regulation of signaling $(\mathrm{ES}=3.5)$, central nervous system development ( $\mathrm{ES}=3.07)$. These findings suggest that alteration of these enriched functions contribute to the expression of RLS.

\section{Replication study}

To confirm the results of the present GWA study, we performed a case-control association analysis for RLS within independent samples that were collected from our research centers. We selected $\mathrm{rs} 11645604\left(\mathrm{OR}=1.531 ; \mathrm{p}=1.18 \times 10^{-6}\right)$ in MPHOSPH6 on chromosome 16q23.3, rs1918752 (OR= 0.6582; $\left.\mathrm{p}=1.93 \times 10^{-6}\right)$, and $\mathrm{rs} 9390170\left(\mathrm{OR}=0.6778 ; \mathrm{p}=7.67 \times 10^{-6}\right)$ in UTRN on chromosome $6 \mathrm{q} 24$ on the basis of the present GWA study findings that showed the strongest association. Seven SNPs (rs6710341 and rs2300478 in MEIS1, rs9357271, rs3923809, and rs9296249 in BTBD9, rs4626664 and rs1975197 in PTPRD), which are known to be correlated with RLS according to previous GWA study findings, were also selected within the replication phase. ${ }^{13-15}$ Among SNPs that were selected from the present and previous GWA study findings, rs9390170 in UTRN (OR=1.6216; $\mathrm{p}=0.0356$ ) showed the most significant association in the recessive model and rs3923809 and rs9296249 in BTBD9 (OR=1.3119; $\mathrm{p}=0.0452, \mathrm{OR}=1.3028$; $\mathrm{p}=0.0463$, respectively) in the allele model as shown in Table 2. From the replication association study, we confirmed the newly found locus rs9390170 in UTRN as a possible novel gene for RLS. We also reconfirmed rs3923809 and rs9296249

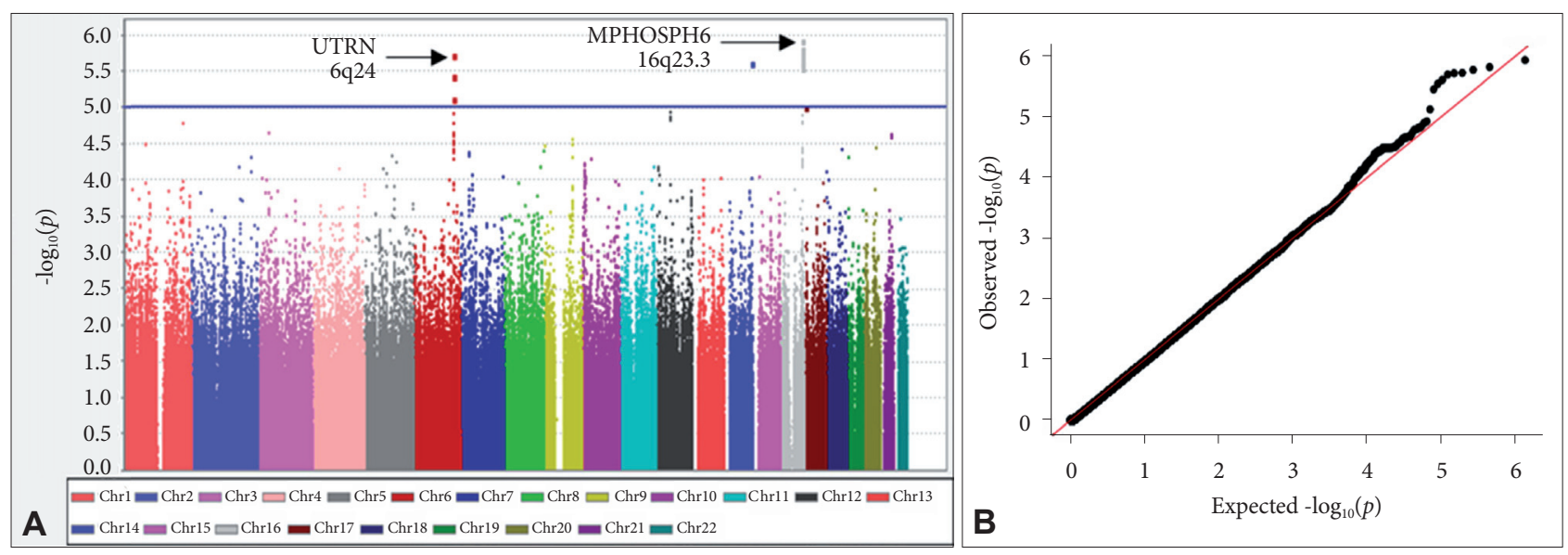

Figure 1. Manhattan plot of genome-wide association results for RLS susceptibility loci and Q-Q plot. In the Manhattan plot (A), the chromosomal position is shown along the $x$-axis, the -log ( $p$-value) for each SNP is shown along the $y$-axis. Horizontal line indicates the $p<10^{-5}$ threshold. Q-Q plot for observed $p$-value for $1,031,877$ SNPs against expected values (B). 

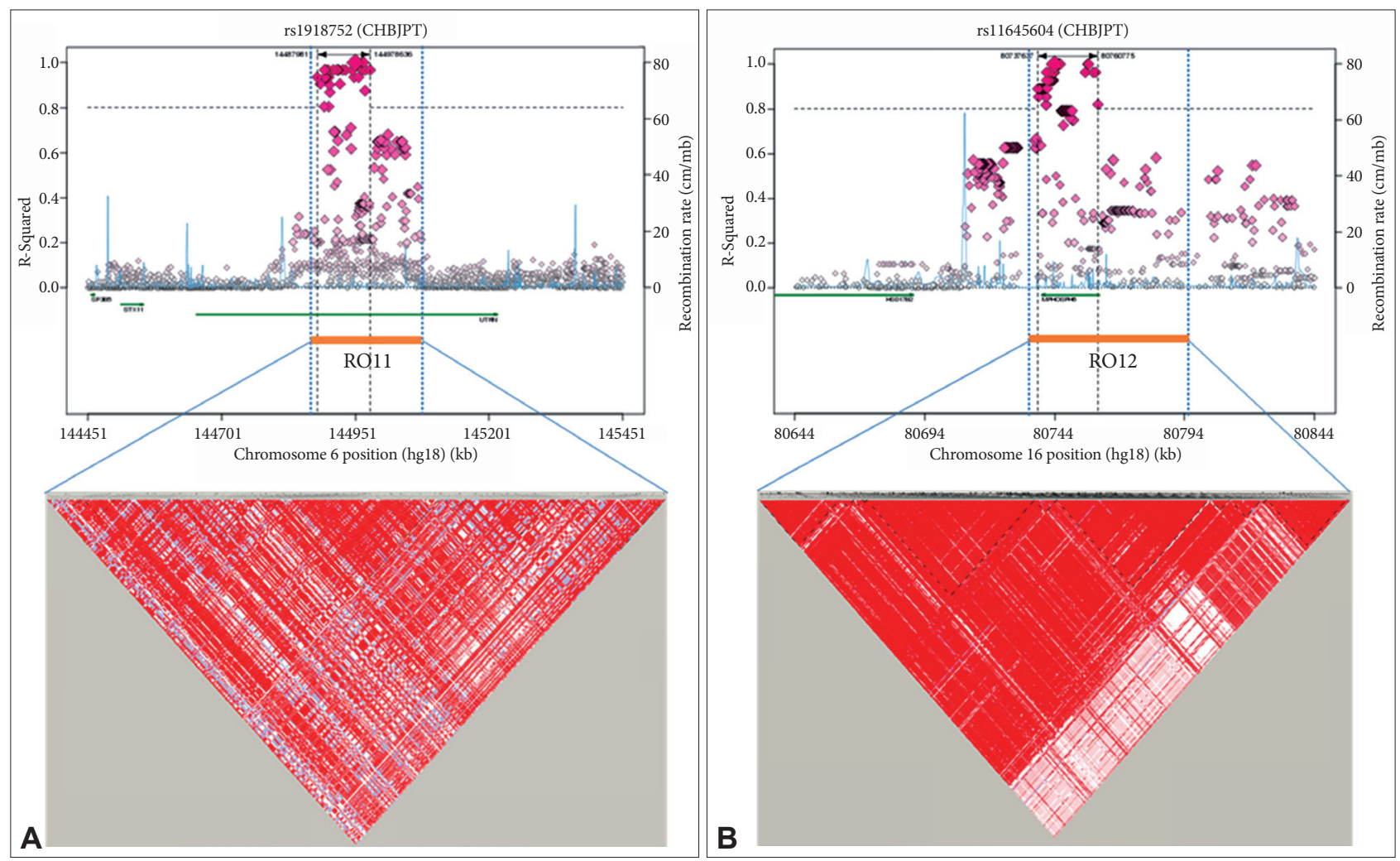

Figure 2. Haplotype association plots for the two ROls in the case-control analysis of GWA. Proxy SNPs with regional recombination rates in the two ROls are shown as determined by SNAP with HapMap 1000 Genomes Pilot 1 data (A and B, top). The regional LDs around the two ROls are shown by Haploview using 1000 Genomes Phase3 JPT+CHB data (A and B, bottom). The D' scores for each paired SNP are indicated by the color scheme. The recombination rate (pale blue line) and ROI span (dotted vertical line) are indicated.

Table 1. The enrichment findings of the functional enrichment analysis within imputed data of GWA study

\begin{tabular}{|c|c|c|c|}
\hline Category & Term & Gene count & $\mathrm{p}$ value \\
\hline Annotation cluster 1 & Enrichment score: 4.19 & & \\
\hline GOTERM_BP_FAT & GO:0007399-nervous system development & 154 & $1.13 \mathrm{E}-08$ \\
\hline GOTERM_BP_FAT & GO:0048699-generation of neurons & 111 & $1.49 \mathrm{E}-07$ \\
\hline GOTERM_BP_FAT & GO:0022008-neurogenesis & 113 & $5.53 \mathrm{E}-07$ \\
\hline GOTERM_BP_FAT & GO:0030182-neuron differentiation & 102 & $8.89 \mathrm{E}-07$ \\
\hline GOTERM_BP_FAT & GO:0048666-neuron development & 88 & $9.23 \mathrm{E}-07$ \\
\hline GOTERM_BP_FAT & GO:0048468-cell development & 138 & $3.05 \mathrm{E}-06$ \\
\hline GOTERM_BP_FAT & GO:0030030-cell projection organization & 101 & $3.48 \mathrm{E}-06$ \\
\hline Annotation cluster 2 & Enrichment score: 3.49 & & \\
\hline GOTERM_BP_FAT & GO:0023051-regulation of signaling & 168 & $1.61 \mathrm{E}-05$ \\
\hline GOTERM_BP_FAT & GO:0009966-regulation of signal transduction & 143 & $2.77 \mathrm{E}-04$ \\
\hline GOTERM_BP_FAT & GO:0010648-negative regulation of cell communication & 75 & $3.86 \mathrm{E}-04$ \\
\hline GOTERM_BP_FAT & GO:0023057-negative regulation of signaling & 74 & $4.69 \mathrm{E}-04$ \\
\hline GOTERM_BP_FAT & GO:0048585-negative regulation of response to stimulus & 82 & $6.40 \mathrm{E}-04$ \\
\hline Annotation cluster 3 & Enrichment score: 3.07 & & \\
\hline GOTERM_BP_FAT & GO:0007417-central nervous system development & 64 & $7.64 \mathrm{E}-05$ \\
\hline GOTERM_BP_FAT & GO:0060322-head development & 54 & $8.70 \mathrm{E}-05$ \\
\hline GOTERM_BP_FAT & GO:0021537-telencephalon development & 23 & $5.47 \mathrm{E}-04$ \\
\hline GOTERM_BP_FAT & GO:0030900-forebrain development & 31 & $6.69 \mathrm{E}-04$ \\
\hline GOTERM_BP_FAT & GO:0007420-brain development & 48 & 8.42E-04 \\
\hline
\end{tabular}

GO: gene ontology 
Table 2. The results of case-control association analysis of SNPs between patients with RLS and healthy controls based on the results of present and previous GWA studies

\begin{tabular}{|c|c|c|c|c|c|c|c|c|c|c|c|}
\hline \multirow[b]{2}{*}{ SNPs } & \multicolumn{3}{|c|}{ Recessive model } & \multicolumn{3}{|c|}{ Dominant model } & \multicolumn{2}{|c|}{ Genotype model } & \multicolumn{3}{|c|}{ Allele model } \\
\hline & $\begin{array}{l}\text { Chi- } \\
\text { square }\end{array}$ & $\mathrm{p}$-value & $\begin{array}{l}\text { Odds } \\
\text { ratio }\end{array}$ & $\begin{array}{l}\text { Chi- } \\
\text { square }\end{array}$ & $\mathrm{p}$-value & $\begin{array}{c}\text { Odds } \\
\text { ratio }\end{array}$ & $\begin{array}{l}\text { Chi- } \\
\text { square }\end{array}$ & $\mathrm{p}$-value & $\begin{array}{l}\text { Chi- } \\
\text { square }\end{array}$ & $\mathrm{p}$-value & $\begin{array}{c}\text { Odds } \\
\text { ratio }\end{array}$ \\
\hline MPHOSPH6 rs11645604 & 0.2514 & 0.6161 & 0.8734 & $6.98 \times 10^{-3}$ & 0.9334 & 0.9844 & 0.2623 & 0.8771 & 0.1024 & 0.749 & 1.0455 \\
\hline UTRN rs9390170 & 4.4148 & $0.0356^{*}$ & 1.6216 & 0.1323 & 0.716 & 1.0796 & 4.5205 & 0.1043 & 2.1077 & 0.1466 & 1.2126 \\
\hline UTRN rs1918752 & 0.2524 & 0.6154 & 1.1205 & 1.7281 & 0.1887 & 0.7588 & 2.7028 & 0.2589 & 0.2897 & 0.5904 & 1.074 \\
\hline MEIS1 rs6710341 & 0.0447 & 0.8326 & 1.0818 & 0.0322 & 0.8576 & 1.0343 & 0.0596 & 0.9706 & 0.0527 & 0.8185 & 1.0352 \\
\hline MEIS1 rs2300478 & 0.3039 & 0.5814 & 0.8087 & 0.3255 & 0.5683 & 0.8983 & 0.4915 & 0.7821 & 0.4527 & 0.5011 & 0.9037 \\
\hline BTBD9 rs9357271 & 1.1432 & 0.285 & 0.5506 & 0.0274 & 0.8686 & 1.0335 & 1.3461 & 0.5101 & 0.0383 & 0.8448 & 0.9669 \\
\hline BTBD9 rs3923809 & 2.8352 & 0.0922 & 1.5357 & 2.4853 & 0.1149 & 1.3586 & 3.9736 & 0.1371 & 4.0101 & $0.0452^{*}$ & 1.3119 \\
\hline BTBD9 rs9296249 & 2.6156 & 0.1058 & 0.6964 & 2.7135 & 0.0995 & 0.7041 & 4.0111 & 0.1346 & 3.9708 & $0.0463^{*}$ & 1.3028 \\
\hline PTPRD rs 1975197 & 0.1863 & 0.666 & 1.1195 & 1.3053 & 0.2532 & 0.8002 & 2.0082 & 0.3664 & 0.3255 & 0.5683 & 1.0804 \\
\hline PTPRD rs4626664 & 2.0055 & 0.1567 & 0.6855 & 0.3075 & 0.5792 & 0.8993 & 2.0123 & 0.3656 & 1.2737 & 0.2591 & 0.8568 \\
\hline
\end{tabular}

${ }^{*} \mathrm{p}<0.05$. SNP: single-nucleotide polymorphism, RLS: restless legs syndrome

Table 3. The results of case-control haplotype analysis of rs9357271/rs3923809/rs9296249 in BTBD9

\begin{tabular}{lcccc}
\hline \multirow{2}{*}{ Haplotype } & Overall p-value & \multicolumn{2}{c}{ Haplotype frequencies } & \multirow{2}{*}{ Permutation p-value } \\
\cline { 3 - 4 } C-G-C & $6.45 \mathrm{E}-03^{*}$ & Patients with RLS & Healthy controls & $7.00 \mathrm{E}-03^{*}$ \\
C-A-T & $3.17 \mathrm{E}-09^{*}$ & 0.3803 & 0.2949 & $0^{*}$ \\
C-G-T & $2.62 \mathrm{E}-05^{*}$ & 0.3171 & 0.1511 & 0 \\
T-A-C & 0.2871 & 0.1678 & 0.2843 & 0.381 \\
T-G-C & 0.8223 & 0.0506 & 0.0672 & 0.829 \\
C-A-C & $5.69 \mathrm{E}-12^{*}$ & 0.0681 & 0.0644 & $1.00 \mathrm{E}-03^{*}$ \\
All haplotypes & $5.69 \mathrm{E}-18^{*}$ & 0.0162 & 0.1381 & $1.00 \mathrm{E}-03^{*}$ \\
\hline
\end{tabular}

${ }^{*} \mathrm{p}<0.05$. RLS: restless legs syndrome

in BTBD9, which were the results of a previous GWA study of RLS from a Western population, ${ }^{13,15}$ as possible susceptibility genes for RLS in a Korean population. For further evaluation, we performed haplotype analysis for selected loci as above, and rs9357271/rs3923809/rs9296249 (overall p=5.69× $10^{-18}$, permutation $\mathrm{p}=0.001$ ) in BTBD9 showed significant associations as shown in Table 3. In the replication phase performed in a Korean population, the results are partially consistent with those of the previous GWA study. ${ }^{13-15}$

\section{DISCUSSION}

The KARE project has been conducted to identify genetic factors associated with various diseases and traits in Korea. We performed a GWA study for RLS within the KARE project, and confirmed the results of the present GWA study by performing a replication study based on the results of variant genes identified in the initial GWA study. There were several findings of the GWA study for RLS that are worthy of close attention. However, virtually all previous GWA findings were performed and replicated within Western subject populations, and no GWA studies had been conducted for RLS in Asian populations. It is crucial to note that this is the first GWA study for RLS with a replication study conducted within an Asian population. The reliability of the replication study increased because it confirmed the results of the present GWA study and simultaneously verified the previous GWA study findings.

We found a novel susceptibility locus for RLS, rs9390170 in UTRN, which encodes utrophin, following confirmation by the replication study in a Korean population. The utrophin gene is on chromosome 6 in humans and encodes a 395 $\mathrm{kDa}$ protein with a high degree of amino acid identity with dystrophin. ${ }^{23}$ Utrophin is expressed in almost all tissues, and is preferentially localized to the neuromuscular and myotendinous junctions in skeletal muscle. ${ }^{24}$ Duchenne's muscular dystrophy (DMD) is a severe X-linked recessive disorder characterized by progressive loss of muscular strength and resulting death due to respiratory or cardiac failure/insufficiency. DMD is due to a lack of dystrophin, a membrane cytoskeletal protein. ${ }^{25}$ Utrophin has received a lot of attention due to its function; it can replace dystrophin and alleviate the muscle pathology of DMD. Hence, nearly all of the studies of utrophin so far have been in the context of DMD. ${ }^{25}$ However, a biopsy research showed that the upregulation of utrophin 
expression on the extrajunctional sarcolemma was found in DMD and other muscular disorders including inflammatory myopathies, diabetic neuropathies, spinal muscular atrophies, and minimal change myopathies. ${ }^{26}$ These results imply that the function of utrophin is not limited to DMD, and we can therefore speculate that its function is related to other neuromuscular diseases, including RLS.

Three primary methods to determine the pathophysiology of RLS have typically been performed: 1) localizing the areas of abnormal central nervous system (CNS) function, 2) finding the neurotransmitter systems associated with medications (dopaminergic), and 3) finding the relationship between iron deficiency and RLS. ${ }^{27}$ RLS is classified as a neurological sensorimotor disease, which means that its pathophysiology involves in sensorimotor system. While most of the studies concerning the pathophysiology of RLS have converged on the nervous system, there have been relatively few studies investigating the relationship between the muscular system and RLS. ${ }^{28}$ Furthermore, no studies have investigated the potential relationship between utrophin and RLS. However, we can assume a novel pathophysiology of RLS as a process of the muscular system based on the results of the present study. When we take into consideration that RLS is related to the muscular system as well as the nervous system, the utrophin gene can be potentially considered as a novel susceptibility gene for RLS. Generally, utrophin plays a role as a member of the dystrophin glycoprotein complex (DGC), which comprises five classes of proteins at the neuromuscular junction (NMJ). ${ }^{29}$ We assume that utrophin itself has a role within the NMJ during the pathogenic process of RLS. The DGC is also distributed in non-muscle tissue including the brain, blood-brain barrier, and choroid plexus, and is associated with the acetylcholine receptor and neuronal nitric oxide synthase (nNOS).$^{30}$ Nitric oxide (NO) is known to be involved in various physiologic processes, including vasodilation and neuromodulation. NO may be considered a connecting link between RLS and utrophin in the pathophysiologic process, as NOS is closely related with utrophin. nNOS was suggested as a marker for complete restoration of the DGC including utrophin in skeletal muscle. ${ }^{31} \mathrm{NO}$ induces increased utrophin levels and enhances its membrane localization at the sarcolemma. ${ }^{32}$ In addition, there have been several studies regarding the relationship between NO and utrophin in muscle as well as in non-muscle tissues. ${ }^{33,34}$

Winkelmann et al. ${ }^{35}$ reported an association of variants of the NOS1 (nNOS) gene and RLS in Germans, which suggested a role of the $\mathrm{NO}$ /arginine pathway in the pathogenesis of RLS, whereas this association was not replicated in the Spanish population. ${ }^{36}$ The NOS1 gene was shown to be associated with Parkinson's disease (PD). ${ }^{37} \mathrm{nNOS}$ is thought to modu- late dopaminergic neurotransmission in the striatum, and this is may be related to the pathogenesis of RLS and PD. ${ }^{38}$ Patton et al. ${ }^{39}$ suggested that the hypoxia pathway is activated in multiple cell types in individuals with RLS, and nNOS production has been shown to increase upon hypoxia pathway activation. The hypoxia pathway is also closely related with cellular iron, which is one of the main pathogenic factors in RLS. Interestingly, there was a case report of a nitroglycerin patch usage for the treatment of RLS. ${ }^{40}$

There are several limitations. First, experts did not make a diagnosis of RLS by face-to-face treatment. The questionnaire was used to determine whether or not RLS symptoms were present. Based on our clinical experience, it is important for RLS diagnosis to be identified directly by the clinician. When using a simple subjective questionnaire for RLS, there might be problems of false-positive or -negative because it is difficult for patients to report their RLS symptoms clearly. This study was conducted for a large-scale cohort, and we classified the RLS symptoms group according to the core symptoms of RLS criteria, after exclusion of medical conditions which may show symptoms similar to RLS. Therefore, it should be noted that the results of this study are limited in interpretation. However, since the study has been replicated in different sample, we believe that the results are valuable. Second, the replication sample was relatively small in size. Although it has modest power, subsequent studies with a larger number of samples are needed to increase the statistical power of genetic studies. Third, the significant results from the replication study lose their statistical power after using Bonferroni correction for multiple testing. Although genetic studies performed with sex and age matched healthy controls, the interpretation is limited because it is an uncorrected result for multiple testing.

To our knowledge, this is the first GWA and replication study performed in Asia for RLS. Although there have been no investigations into the direct relationship between utrophin and RLS, we can hypothesize that utrophin is involved in the pathogenesis of RLS, particularly as it relates to the neuromuscular system. Moreover, we anticipate that utrophin can be the link between the distinct known pathogenesis of RLS such as dopamine neurotransmission and iron deficiency. Further investigation into the function and role of utrophin as a causative factor in the pathogenesis of RLS should confirm our findings and hypothesis. Based on the results of the first GWA study and the replication study of RLS in a Korean population taken together with the evidence for a potential relationship between utrophin and RLS, we suggest the existence of a susceptibility locus, rs9390170 on $6 \mathrm{q} 24$, for RLS. 


\section{Supplementary Materials}

The online-only Data Supplement is available with this article at https://doi.org/10.4306/pi.2017.14.6.830.

\section{Acknowledgments}

The GWAS samples were provided with biospecimens and data from Korean Genome Analysis Project (4845-301), the Korean Genome and Epidemiology Study (4851-302), and Korea Biobank Project (4851-307, KBP-2013-000) that were supported by the Center for Disease Control and Prevention, Republic of Korea.

This study was supported by the Korea Health 21 R\&D Project funded by the National Research Foundation of Korea (NRF-2016M3C7A190 4345).

\section{REFERENCES}

1. Allen RP, Picchietti DL, Garcia-Borreguero D, Ondo WG, Walters AS, Winkelman JW, et al. Restless legs syndrome/Willis-Ekbom disease diagnostic criteria: updated International Restless Legs Syndrome Study Group (IRLSSG) consensus criteria--history, rationale, description, and significance. Sleep Med 2014;15:860-873.

2. Innes KE, Selfe TK, Agarwal P. Prevalence of restless legs syndrome in North American and Western European populations: a systematic review. Sleep Med 2011;12:623-634.

3. Allen RP, Walters AS, Montplaisir J, Hening W, Myers A, Bell TJ, et al. Restless legs syndrome prevalence and impact: REST general population study. Arch Intern Med 2005;165:1286-1292.

4. Connor JR, Wang XS, Patton SM, Menzies SL, Troncoso JC, Earley CJ, et al. Decreased transferrin receptor expression by neuromelanin cells in restless legs syndrome. Neurology 2004;62:1563-1567.

5. Trenkwalder C, Paulus W. Restless legs syndrome: pathophysiology, clinical presentation and management. Nat Rev Neurol 2010;6:337-346.

6. Allen RP, Picchietti D, Hening WA, Trenkwalder C, Walters AS, Montplaisi J, et al. Restless legs syndrome: diagnostic criteria, special considerations, and epidemiology. A report from the restless legs syndrome diagnosis and epidemiology workshop at the National Institutes of Health. Sleep Med 2003;4:101-119.

7. Garcia-Borreguero D, Cano-Pumarega I. New concepts in the management of restless legs syndrome. BMJ 2017;356:j104.

8. Barriere G, Cazalets JR, Bioulac B, Tison F, Ghorayeb I. The restless legs syndrome. Prog Neurobiol 2005;77:139-165.

9. Whittom S, Dauvilliers Y, Pennestri MH, Vercauteren F, Molinari N, Petit D, et al. Age-at-onset in restless legs syndrome: a clinical and polysomnographic study. Sleep Med 2007;9:54-59.

10. Winkelmann J, Polo O, Provini F, Nevsimalova S, Kemlink D, Sonka K, et al. Genetics of restless legs syndrome (RLS): state-of-the-art and future directions. Mov Disord 2007;22(suppl 18):S449-S458.

11. Chen S, Ondo WG, Rao S, Li L, Chen Q, Wang Q. Genomewide linkage scan identifies a novel susceptibility locus for restless legs syndrome on chromosome 9p. Am J Hum Genet 2004;74:876-885.

12. Allen RP. Controversies and challenges in defining the etiology and pathophysiology of restless legs syndrome. Am J Med 2007;120:S13-S21.

13. Winkelmann J, Schormair B, Lichtner P, Ripke S, Xiong L, Jalilzadeh S, et al. Genome-wide association study of restless legs syndrome identifies common variants in three genomic regions. Nat Genet 2007;39:10001006.

14. Schormair B, Kemlink D, Roeske D, Eckstein G, Xiong L, Lichtner P, et al. PTPRD (protein tyrosine phosphatase receptor type delta) is associated with restless legs syndrome. Nat Genet 2008;40:946-948.

15. Stefansson H, Rye DB, Hicks A, Petursson H, Ingason A, Thorgeirsson TE, et al. A genetic risk factor for periodic limb movements in sleep. $\mathrm{N}$ Engl J Med 2007;357:639-647.

16. Winkelmann J, Czamara D, Schormair B, Knauf F, Schulte EC, Trenkwalder $\mathrm{C}$, et al. Genome-wide association study identifies novel rest- less legs syndrome susceptibility loci on 2p14 and 16q12.1. PLoS Genet 2011;7:e1002171.

17. Cho YS, Go MJ, Kim YJ, Heo JY, Oh JH, Ban HJ, et al. A large-scale genome-wide association study of Asian populations uncovers genetic factors influencing eight quantitative traits. Nat Genet 2009;41:527534.

18. Ban HJ, Kim SC, Seo J, Kang HB, Choi JK. Genetic and metabolic characterization of insomnia. PLoS One 2011;6:e18455.

19. Howie BN, Donnelly P, Marchini J. A flexible and accurate genotype imputation method for the next generation of genome-wide association studies. PLoS Genet 2009;5:e1000529.

20. Purcell S, Neale B, Todd-Brown K, Thomas L, Ferreira MA, Bender D, et al. PLINK: a tool set for whole-genome association and populationbased linkage analyses. Am J Hum Genet 2007;81:559-575.

21. Huang da W, Sherman BT, Lempicki RA. Bioinformatics enrichment tools: paths toward the comprehensive functional analysis of large gene lists. Nucleic Acids Res 2009;37:1-13.

22. Huang da W, Sherman BT, Lempicki RA. Systematic and integrative analysis of large gene lists using DAVID bioinformatics resources. Nat Protoc 2009;4:44-57.

23. Pearce M, Blake DJ, Tinsley JM, Byth BC, Campbell L, Monaco AP, et al. The utrophin and dystrophin genes share similarities in genomic structure. Hum Mol Genet 1993;2:1765-1772.

24. Helliwell TR, Man NT, Morris GE, Davies KE. The dystrophin-related protein, utrophin, is expressed on the sarcolemma of regenerating human skeletal muscle fibres in dystrophies and inflammatory myopathies. Neuromuscul Disord 1992;2:177-184.

25. Galvagni F, Cantini M, Oliviero S. The utrophin gene is transcriptionally up-regulated in regenerating muscle. J Biol Chem 2002;277:1910619113.

26. Lukas Z. The up-regulation of utrophin is not limited to muscular dystrophies. Gen Physiol Biophys 1999;18(Suppl 1):87-89.

27. Allen RP, Earley CJ. Restless legs syndrome: a review of clinical and pathophysiologic features. J Clin Neurophysiol 2001;18:128-147.

28. Wahlin-Larsson B, Ulfberg J, Aulin KP, Kadi F. The expression of vascular endothelial growth factor in skeletal muscle of patients with sleep disorders. Muscle Nerve 2009;40:556-561.

29. Blake DJ, Weir A, Newey SE, Davies KE. Function and genetics of dystrophin and dystrophin-related proteins in muscle. Physiol Rev 2002; 82:291-329.

30. Haenggi T, Fritschy JM. Role of dystrophin and utrophin for assembly and function of the dystrophin glycoprotein complex in non-muscle tissue. Cell Mol Life Sci 2006;63:1614-1631.

31. Wells KE, Torelli S, Lu Q, Brown SC, Partridge T, Muntoni F, et al. Relocalization of neuronal nitric oxide synthase (nNOS) as a marker for complete restoration of the dystrophin associated protein complex in skeletal muscle. Neuromuscul Disord 2003;13:21-31.

32. Chaubourt E, Fossier P, Baux G, Leprince C, Israël M, De La Porte S. Nitric oxide and l-arginine cause an accumulation of utrophin at the sarcolemma: a possible compensation for dystrophin loss in duchenne muscular dystrophy. Neurobiol Dis 1999;6:499-507.

33. Prochniewicz E, Henderson D, Ervasti JM, Thomas DD. Dystrophin and utrophin have distinct effects on the structural dynamics of actin. Proc Natl Acad Sci U S A 2009;106:7822-7827.

34. Adams ME, Anderson KN, Froehner SC. The alpha-syntrophin PH and PDZ domains scaffold acetylcholine receptors, utrophin, and neuronal nitric oxide synthase at the neuromuscular junction. J Neurosci 2010;30:11004-11010.

35. Winkelmann J, Lichtner P, Schormair B, Uhr M, Hauk S, Stiasny-Kolster $\mathrm{K}$, et al. Variants in the neuronal nitric oxide synthase (nNOS, NOS1) gene are associated with restless legs syndrome. Mov Disord 2008; 23:350-358.

36. Jimenez-Jimenez FJ, Alonso-Navarro H, Martinez C, Zurdo M, TurpinFenoll L, Millan-Pascual J, et al. Neuronal nitric oxide synthase (nNOS, NOS1) rs693534 and rs7977109 variants and risk for restless legs syn- 
drome. J Neural Transm (Vienna) 2015;122:819-823.

37. Rife T, Rasoul B, Pullen N, Mitchell D, Grathwol K, Kurth J. The effect of a promoter polymorphism on the transcription of nitric oxide synthase 1 and its relevance to Parkinson's disease. J Neurosci Res 2009;87: 2319-2325.

38. West AR, Galloway MP, Grace AA. Regulation of striatal dopamine neurotransmission by nitric oxide: effector pathways and signaling mechanisms. Synapse 2002;44:227-245.

39. Patton SM, Ponnuru P, Snyder AM, Podskalny GD, Connor JR. Hypoxia-inducible factor pathway activation in restless legs syndrome patients. Eur J Neurol 2011;18:1329-1335.

40. Ulfberg J. Treatment of restless legs syndrome with a nitroglycerin patch. Sleep Med 2012;13:1326. 